\title{
Greedy Based Proactive Spectrum Handoff Scheme for Cognitive Radio Systems
}

\author{
Zhengjia $\mathrm{Xu}$ \\ School of Aerospace, \\ Transport and Manufacturing \\ Cranfield University \\ MK43 0AL, UK \\ Zhengjia.Xu@cranfield.ac.uk
}

\author{
Ivan Petrunin \\ School of Aerospace, \\ Transport and Manufacturing \\ Cranfield University \\ MK43 0AL, UK \\ i.petrunin@cranfield.ac.uk
}

\author{
Teng Li \\ School of Aerospace, \\ Transport and Manufacturing \\ Cranfield University \\ MK43 0AL, UK \\ tengli@ cranfield.ac.uk
}

\author{
Antonios Tsourdos \\ School of Aerospace, \\ Transport and Manufacturing \\ Cranfield University \\ MK43 0AL, UK \\ a.tsourdos@cranfield.ac.uk
}

\begin{abstract}
The aeronautical spectrum becomes increasingly congested due to raising number of non-stationary users, such as unmanned aerial vehicles (UAVs). With the growing demand to spectrum capacity, cognitive radio technology is a promising solution to maximize the utilization of spectrum by enabling communication of secondary users (SUs) without interfering with primary users (PUs). In this paper we formulate and solve a multi-parametric objective function for proactive handoff scheme in multiple input multiple output (MIMO) system constrained by QoS requirements. To improve the efficiency of handoff scheme for multiple communicating UAVs the greedy strategy is adopted. An innovative aspect of our solution includes consideration of quality of service (QoS) components, e.g. opportunistic service time, channel quality, etc. Some of these components, for example collision probability and false alarm probability, affect QoS in a negative way and are considered as constraints. Simulation of handoff scheme has been performed to evaluate the performance of the proposed algorithm in selecting multiple channels when the spectrum environment changes. The performance of handoff scheme is compared with random selection method and is found outperforming the random selection method in terms of averaged utilization ratio. Analysis of results has shown that the spectrum utilization ratio can be doubled by considering wider bandwidth (more channels) and by making QoS requirements less strict. In both cases this leads to near-linear increase in time consumption for handoff scheme generation.
\end{abstract}

Index Terms-proactive handoff, UAV communication, spectrum mobility, cognitive radio, greedy theory

\section{INTRODUCTION}

Communication paradigm of Unmanned Traffic Management (UTM) system assumes large number of users involved in the communication process. Some of these users will be owning communication channels, i.e. the primary users (PUs), while others, the secondary users (SUs), can only use the communication channel when it is not used by PUs. Without owning the communication channel in congested spectrum environment, the communication between UAVs and ground stations (e.g. UTM system) becomes challenging. Under the concept of cognitive radio (CR), the SUs are permitted to opportunistically occupy spectrum vacancies in communication channels of PUs, which is beneficial for maximizing the spectrum utilization.
The architecture of cognitive communication with handoff for UTM scenario is illustrated in Fig. 1. The communication handoff scheme is generated according to preliminary analyzed RF environment and is updated periodically during the whole duration of UAV flight. Each UAV is equipped with multiple transmitters to make it agile in communication sense and capable of dynamic access to spectrum by switching channels or frequency bands, achieving thus higher throughput.

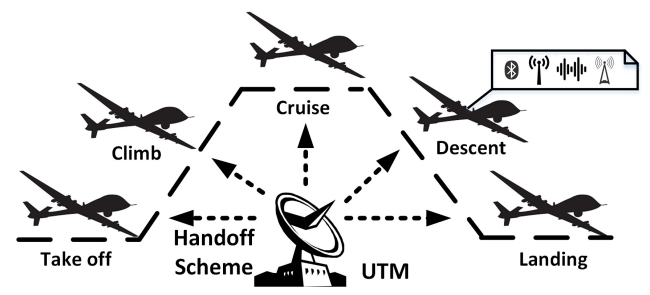

Fig. 1. Handoff scheme for UTM scenario

Due to the efficiency of greedy strategy, several studies have been done on spectrum handoff schemes implementing greedy algorithms, e.g. auction and greedy based spectrum allocation in multiple bands [1], quadratic greedy approach and handoff scheme based on knapsack model for multichannel selection and access [2], greedy and partially observable Markov decision process (POMDP) approaches for maximizing the throughput capacity [3]. Work [4] investigates and compares the performance of three greedy based schemes: $\varrho^{P R E}, \mathrm{SL}(\mathrm{K}), k_{t} h$-UCB 1 for decentralized blind spectrum selection. In addition, [5] proposes a spectrum access protocol based on greedy channel selection and single rendezvous (SRV) coordination scheme for avoiding collision.

Generally, the spectrum handoff schemes can be categorized into two types: reactive and proactive [6]. The proactive handoff scheme indicates that the handoff action is performed based on the prediction of PU traffic model before the triggering event occurs, while the reactive handoff is performed without having knowledge of PU models and is triggered re-actively after encountering the event. The proactive handoff method outperforms the reactive one in terms of latency when the sensing time is large [7]. However, when prediction of the PU 
traffic fails to describe the practical scenario, the proactive handoff leads to a poor solution [8].

Several studies have been done concerning proactive handoff schemes. Work [9] proposes a preemptive resume priority (PRP) $\mathrm{M} / \mathrm{G} / 1$ queuing model to evaluate and minimize total service time for multiple spectrum handoffs. Protocol for collision reduction is proposed in [10] as well as a distributed channel selection algorithm that uses Markov chain for channel selection. A comprehensive survey of handoff schemes for cognitive radio is carried out in [7].

This paper proposes an efficient proactive handoff scheme with considerations of multiple input multiple output (MIMO) communication system under constraints. We introduce objective function for improving communication behaviour over multiple channels in terms of available quality of service (QoS) while considering also the negative effects of PUs transmission disruption. The handoff scheme for multi-channel communication is performed by adopting greedy policy strategy together with an adaptive threshold to accelerate the computation.

\section{HANDOFF SCHEME MODEL}

The handoff is usually considered as a single event in timefrequency domain and the handoff scheme is the group of single handoff events. Fig. 2 demonstrates the handoff scheme for two transmitters $C R_{1}$ and $C R_{2}$ at the same UAV. Here $\mathcal{T}$ denotes our desired handoff solution consisting of a set of potential spectrum opportunities in multiple channels. The purpose of handoff scheme is to create the handoff solution $\mathcal{T}_{a}$ for each $\mathrm{CR}$ in order to switch between channels and to maximize the utilization of vacant frequency opportunities.

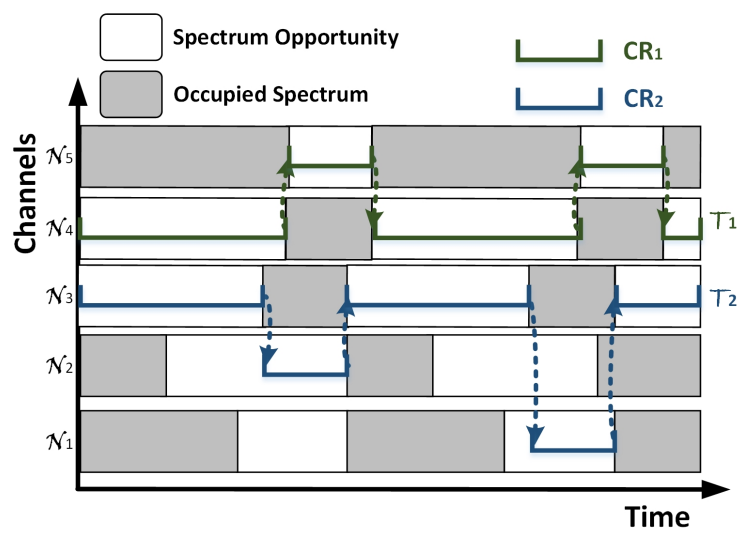

Fig. 2. Concept of handoff scheme

In the design process the following assumptions were made:

1) Different CRs can not occupy the same spectrum opportunity.

2) When accessing spectrum opportunity, CRs cannot switch channels before finishing the occupation of current opportunity.

3) CRs can access the spectrum in the middle of spectrum opportunity.

4) CRs cannot switch back and utilize the past spectrum opportunity.
5) Latencies of spectrum sensing and spectrum probing are not considered.

Several aspects of QoS are considered in this case: opportunistic service time, quality of channel, available channels, set of CRs, collision probability idle and false alarm probability. Besides, the energy balance is also introduced for power allocation purpose. The meaning of the QoS components and some assumptions associated with them are discussed below.

Opportunistic service time $T_{d}$ describes the duration of spectrum opportunity and affects the density of vacant opportunities. It is easy to see that frequency opportunities with longer service time will be utilized first of all as this minimizes the number of single handoff events.

Quality of channel $Q$ is a parameter describing the degradation of signal quality that is based on a theoretical channel model. In this study we utilize historical measurement of channel quality that is also assumed to be constant within the given period of time. Implementation of the channel quality update according to the dynamically changing environment is the topic of the future work.

Available channels $\mathcal{N}$ will influence the number of vacant opportunities $\mathcal{T}$. With larger number of channels $\mathcal{N}$, it is predicted that CRs will have higher probability to find spectrum opportunities for transmission.

The set of CRs $\mathcal{A}$ represents the number of CRs. CR system throughput capacity increases with larger number of $\mathcal{A}$.

Because of the fact that other SUs may exist in other system and may potentially occupy the same channel, the collision probability $P_{c}$ is introduced here to describe the chance that the channel can be accessed by other SUs interfering with each other.

Probability of channel idle $P_{i}$ is derived from the confidence on the availability of each channel during the spectrum sensing period. This probability should be minimized at the decision stage of handoff.

False alarm probability $P_{f}$ describes the accuracy of detection at the spectrum sensing stage and should be minimized during the handoff as well.

To avoid the excessive computation loads and equalize the energy consumption among CRs, the energy balancing functionality is implemented by analysis of the total transmission time of each CR after implementation of the handoff scheme.

\section{HANDOFF AlgORITHM}

Handoff algorithm development is based on three principles:

1) Channel allocation starts with selecting spectrum opportunity that is closest to the current opportunity in the time domain; 2) Channels with poor QoS will be avoided in the handoff action; 3) The spectrum opportunities with longer duration have more chances to be selected.

Based on above principles, the differential objective function for the handoff scenario is proposed as follows:

$$
\Delta f_{a}\left(j \mid \mathcal{T}_{a}\right)=T_{d}^{j} Q^{j} P_{i}^{j} \lambda_{T_{m}}^{\Delta T_{m}{ }_{a}^{j}} \lambda_{c}^{P_{c}^{j}} \lambda_{f}^{P_{f}^{j}} \lambda_{n}^{\tau\left(\mathcal{P}_{a}^{j}\right)}
$$

where $\left(j \mid \mathcal{T}_{a}\right)$ represents the $j_{t h}$ spectrum opportunity based on $\mathcal{T}_{a}$ result; $\Delta T_{m}$ represents the time interval between 
current time and time of the next available opportunity $j_{a}$; $\mathcal{P}_{a}^{j}$ represents total transmission time of $a_{t h} \mathrm{CR}$ towards the $j_{\text {th }}$ spectrum opportunity according to the allocated spectrum opportunities. $\lambda_{T_{m}}, \lambda_{c}, \lambda_{f}$ and $\lambda_{n}$ are the discount factors describing the service time, collision probability, false alarm probability and energy balance, respectively; $\tau$ is the function of accumulated service time. $\lambda_{c}, \lambda_{f}$ and $\lambda_{n}$ are also considered as constraint factors since these QoS factors give negative effect on the objective function. The values of $\lambda$ are bounded by the range of $(0,1]$ to ensure the objective function decreases monotonically.

The objective function (1) can be proved to be monotonic and submodular according to [11] and it is expected to be maximized at each iteration within the scheme.

The developed algorithm of spectrum handoff is a significantly modified version of the centralized greedy algorithm presented in [11]. The algorithm of the proposed handoff scheme is presented below (Algorithm 1).

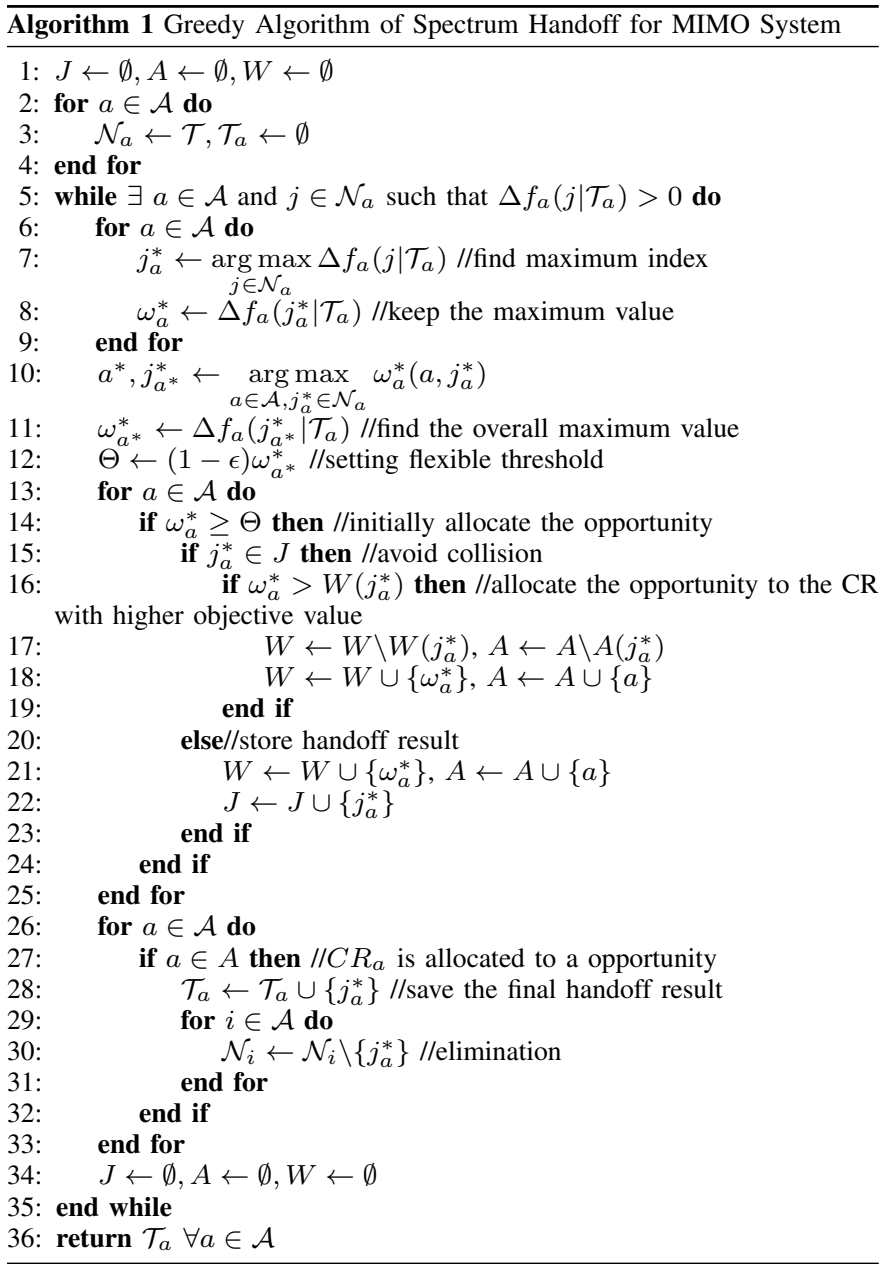

In this algorithm $J, A$ and $W$ denote the set of spectrum opportunities that are used for collision avoid in the allocation process, index of channel and objective value respectively; $\mathcal{N}_{a}$ represents the $a_{t h}$ CR's available spectrum opportunities; $\Theta$ is the threshold of objective function that is being adapted at each iteration with $\epsilon$ as its weight; $W \backslash W\left(j_{a}^{*}\right)$ represents removal of the element in $W$ with index of $j_{a}^{*}$. Symbol $\cup$ denotes addition of a new element in the original set.

After the initialization of parameters, the $a_{t h} \mathrm{CR}$ is assigned with $\mathcal{T}$ spectrum opportunities.

At the next step, within the main loop, the maximum value of objective function is calculated for each CR. The index of the maximum of the objective function is assigned to $j_{a}^{*}$ and the corresponding value assigned to $\omega_{a}^{*}$. After that we calculate the adaptive threshold $\Theta$ according to the maximum value stored in $\omega_{a}^{*}$. If the maximum of the objective function for $a_{t h} \mathrm{CR}$ is larger than threshold, this CR can be potentially assigned with spectral opportunity at this iteration. In that case, verification of collision absence is required, i.e. collision avoidance needs to be performed. The collision may occur when another CR occupies the same opportunity, and in this case we resolve the conflict by reassigning the spectrum opportunity to the $\mathrm{CR}$ with larger objective function value. If the value of the objective function is less than the threshold, the handoff is not performed for $a_{t h} \mathrm{CR}$ at this iteration.

When $a_{t h} \mathrm{CR}$ is confirmed to be allocated with spectrum opportunity, i.e. the handoff is successful, the result will be stored in $\mathcal{T}_{a}$. This spectrum opportunity is then removed from $\mathcal{N}$ to prevent being repeatedly utilized by other CRs. Clearing parameters (i.g. $A, W$ and $J$ ) is necessary at each iteration.

The allocation process in this handoff scheme iterates until all required resources are allocated to CRs. The stop condition can also be set according to a maximum time limit.

It should be mentioned that only the allocated spectrum opportunities $j_{a}^{*}$ have been removed from the resource set $\mathcal{N}$ according to line 30 , while the eliminations can also be performed in time domain to reduce the size of the available resources $\mathcal{N}$. For further reduction of the computation time $\mathcal{N}_{a}$ can be assigned to the closest to $a_{t h} \mathrm{CR}$ spectrum opportunity.

\section{Results AND Discussion}

Simulation is performed for the case when 3 CRs need to be allocated with spectrum opportunities in 5 channels. The spectrum opportunity map is randomly generated based on Monte Carlo method; the opportunities are periodic in time domain. The discount factors $\lambda_{T_{m}}, \lambda_{c}, \lambda_{f}$ and $\lambda_{n}$ are set as $0.6,0.6,0.5$ and 0.4 respectively. The threshold weight $\epsilon$ is 0.2 . Other configuration values of QoS aspects among 5 channels are presented in Table. I.

TABLE I

SIMULATION PARAMETERS

\begin{tabular}{llllll}
\hline & Channel 1 & channel 2 & channel 3 & channel 4 & channel 5 \\
\hline$T_{d}$ & 0.1181 & 0.1905 & 0.1823 & 0.1674 & 0.1568 \\
$Q$ & 0.8883 & 0.5297 & 0.0087 & 0.4737 & 0.9537 \\
$P_{c}$ & 0.0082 & 0.2320 & 0.2357 & 0.3324 & 0.7173 \\
$P_{i}$ & 0.7473 & 0.5466 & 0.7483 & 0.7469 & 0.0297 \\
$P_{f}$ & 0.1628 & 0.2710 & 0.2722 & 0.3250 & 0.1846 \\
\hline
\end{tabular}

The handoff result displayed in Fig. 3 is obtained for simulation time of $1 \mathrm{~s}$. Here we use channel instead of spectrum frequency when plotting the results. The grey squares in the plots represent spectrum opportunities. Three colour 


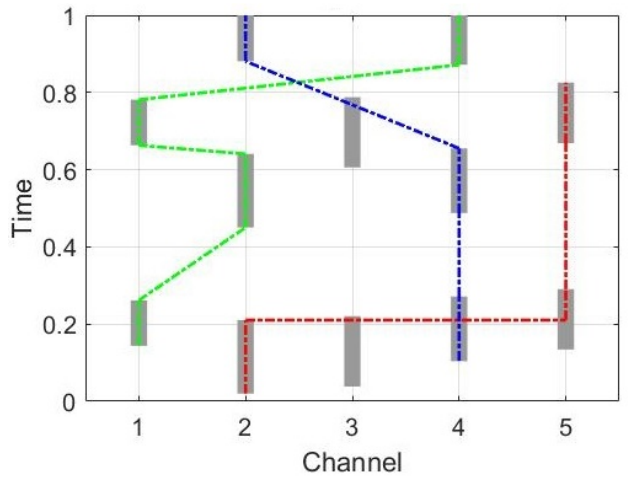

(a) $\lambda_{c}=0.6$

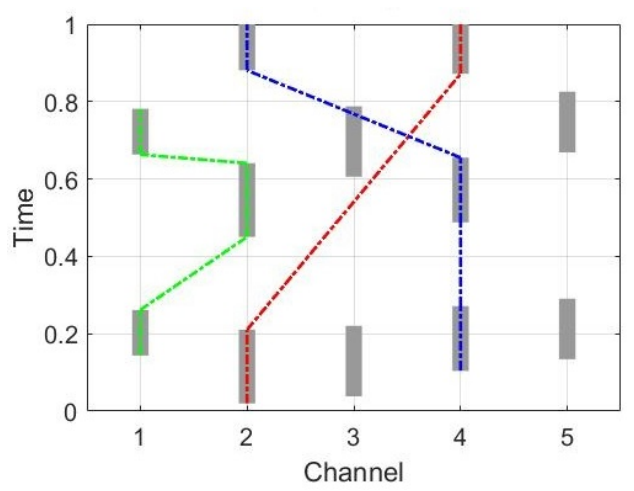

(b) $\lambda_{c}=0.5$

Fig. 3. Handoff simulation restrained by collision probability

lines show the handoff scheme for each CR over five available channels. As it is displayed in Fig. 3(a), when $\lambda_{c}=0.6$ all the opportunities of all channels (except channel 3 , which has lowest quality $Q=0.0087$ among the five channels) have been allocated to CRs. The behaviour of "Blue" CR that switches from channel 4 to 2 at the time of $0.88 \mathrm{~s}$ instead of staying in the same channel can be improved either by considering global maximization that includes all opportunities or by introducing handoff latency.

Considering the high value of collision probability $P_{c}$ (0.7173) in channel 5 from Table. I, the elimination of channel 5 from handoff scheme can be achieved by decreasing $\lambda_{c}$ value from 0.6 to 0.5. The handoff result is presented in Fig. 3(b). It can be seen that the channel 5 is not considered in such case, which means the channel selection result is affected by leveraging values between discount factors.

Additionally, in this case spectrum opportunity in channel 4 at $0.88 \mathrm{~s}$ is allocated to the "Red" CR instead of the "Green" one, because "Green" CR has been allocated more spectrum opportunities than the "Red" CR. One can see from this example that energy balancing is also possible in this way by leveraging load between CRs.

The performance of the proposed handoff scheme can be demonstrated by considering the utilization ratio, i.e. how well the scheme utilizes $N$ channels in the analyzed frequency band in CRs allocation process.
For this purpose we simulate the case of larger number of CRs: $N=10$. The overall constraint factor is defined as a sum of individual constraint factors: $K=\lambda_{c}+\lambda_{f}+\lambda_{n}$. The reference value $K_{\text {ref }}$ is obtained using $\lambda_{T_{m}}=0.7, \lambda_{c}=0.2$, $\lambda_{f}=0.15$ and $\lambda_{n}=0.5$. Simulation time $T_{s}$ is set to $1 \mathrm{~s}$. Based on results of generated handoff scheme, we define the utilization ratio as $R=\left(\sum_{i=1}^{N} \mathcal{T}_{i}\right) /\left(N T_{s}\right)$, where $N$ is the number of CRs.

During the simulation we randomly generate maps of spectrum opportunities, create handoff scheme and calculate utilization ratio $R$. Having this procedure repeated large number of times, we can calculate the averaged utilization ratio as follows: $\bar{R}=\left(\sum_{i=1}^{N_{l}} R_{N_{l}}\right) / N_{l}$, where $N_{l}$ is the number of repetitions of handoff scheme calculation, which was set to 800. Value of $\bar{R}$ is calculated for a range of overall constraint factor $K$ as a function of channel number $N$ and is presented in Fig. 4. Same configuration was adopted for analyzing the time consumption with results shown in Fig. 5.

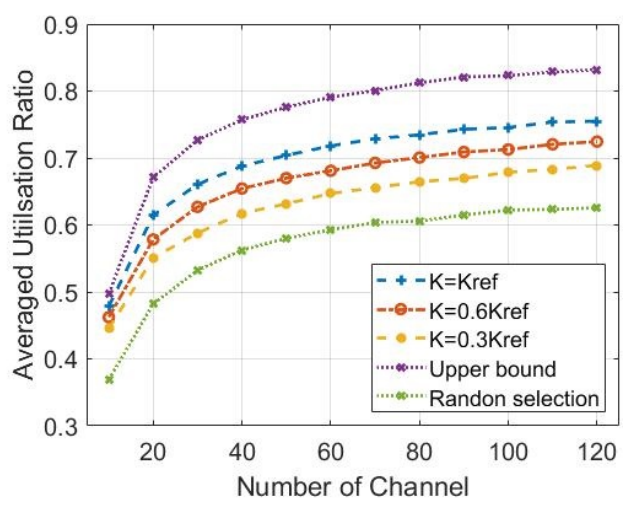

Fig. 4. Comparison of averaged utilization ratio $\bar{R}$ vs number of channel $N$ with different $K$

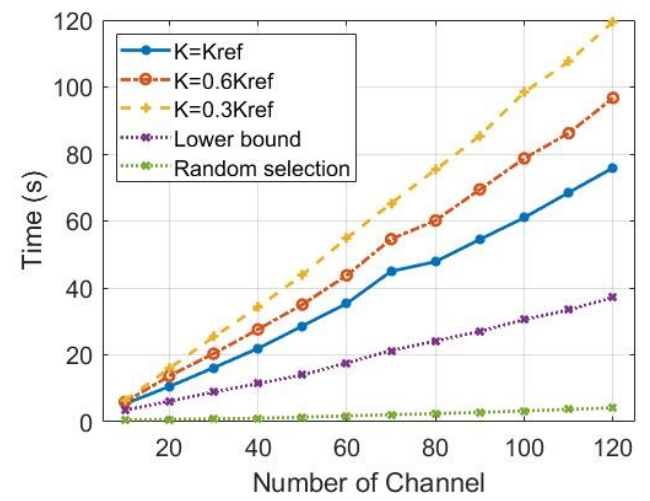

Fig. 5. Comparison of the time consumption vs number of channel $N$ with different $K$

When the individual constraints $\lambda_{c}, \lambda_{f}$ and $\lambda_{n}$ are set to their maximum value of 1 , meaning that they are invalid in this case the utilization ratio is reaching the maximum level (shown as the upper bound in Fig. 4) and the time consumption 
is expected to be minimized (shown as the lower bound in Fig. 5). We consider above two bounds as the best (suboptimal) reference bounds that can be used for analysis of the constraints values influence on the handoff efficiency.

As it is illustrated in Fig. 4, the proposed handoff scheme is more effective compared to the random channel selection method [10]. The ratio $\bar{R}$ increases when $N$ becomes larger, although the rate of growth is highest at the beginning and then decreases. However, according to Fig. 4, the computation time increases almost linearly with the number of available channels.

Analyzing results in Fig. 4 in terms of $K$ factor we can note that utilization ratio increases for larger values of $K$, i.e. weaker constraints contribute to higher occupation of spectrum, but reduce QoS for the link. Additionally, according to Fig. 5, the weaker constraints also lead to slower increase in computation time when the number of channels increases.

For the purpose of comparison of the proposed handoff scheme with random channel selection scheme the overall constraint factor $K$ was set according to the reference value used previously. Results presented in Fig. 4 and Fig. 5 show that the proposed scheme provides essential increase in performance, but at the cost of increased computational time.

\section{Conclusions}

This paper proposes a proactive handoff scheme for UAV communication with multiple CRs. By adopting greedy strategy in the handoff scheme and considering several constraints for maintaining QoS, the handoff scheme is generated through an iterative process defining an objective function. The handoff scheme was compared with random channel selection method, and effectiveness of handoff was analyzed with respect to changes in constraint factors. Analysis of results has shown that spectrum utilization ratio can be improved by considering wider bandwidth (more channels) and making QoS requirements less strict. In both cases it leads to increase in time consumption for handoff scheme generation.

\section{REFERENCES}

[1] Z. Shi and G. Luo, "Multi-band spectrum allocation algorithm based on first-price sealed auction," Cybernetics and Information Technologies, vol. 17, no. 1, pp. 104-112, 2017.

[2] S. Dahi and S. Tabbane, "Quadratic greedy algorithm for multichannel access in cognitive radio network," International Journal of Computer Applications, vol. 91, 032014.

[3] A. Choudhary and M. Singh, "Opportunistic spectrum access using greedy approach in cognitive radios," International Journal of VLSI \& Signal processing applications, vol. 1, pp. 10-15, 012011.

[4] Y. Chen, H. Zhou, R. Kong, L. Zhu, and H. Mao, "Decentralized blind spectrum selection in cognitive radio networks considering handoff cost," Future Internet, vol. 9, no. 2, p. 10, 2017.

[5] M. Mehrnoush and V. T. Vakili, "Proactive srv spectrum handoff protocol based on gcs scheme in cognitive radio adhoc network," arXiv preprint arXiv:1611.07020, 2016.

[6] L. Wang and C. Wang, "Spectrum handoff for cognitive radio networks: Reactive-sensing or proactive-sensing?" in 2008 IEEE International Performance, Computing and Communications Conference, Dec 2008, pp. 343-348.

[7] K. Kumar, A. Prakash, and R. Tripathi, "Spectrum handoff in cognitive radio networks: A classification and comprehensive survey," Journal of Network and Computer Applications, vol. 61, pp. 161-188, 2016.
[8] L.-C. Wang, C.-W. Wang, and C.-J. Chang, "Modeling and analysis for spectrum handoffs in cognitive radio networks," IEEE Transactions on Mobile Computing, vol. 11, no. 9, pp. 1499-1513, 2012.

[9] C. . Wang and L. . Wang, "Modeling and analysis for proactivedecision spectrum handoff in cognitive radio networks," in 2009 IEEE International Conference on Communications, June 2009, pp. 1-6.

[10] Y. Song and J. Xie, "Performance analysis of spectrum handoff for cognitive radio ad hoc networks without common control channel under homogeneous primary traffic," Proceedings - IEEE INFOCOM, 042011.

[11] H.-S. Shin, T. Li, and P. Segui-Gasco, "Sample greedy based task allocation for multiple robot systems," arXiv preprint arXiv:1901.03258, 2019. 
$2019-11-21$

Greedy based proactive spectrum handoff scheme for cognitive radio systems

Xu, Zhengjia

IEEE

Xu Z, Petrunin I, Li T, Tsourdos A. (2019) Greedy based proactive spectrum handoff scheme for cognitive radio systems. In: 2019 IEEE Cognitive Communications for Aerospace Applications Workshop (CCAAW), 25-26 June 2019, Cleveland, OH, USA https://doi.org/10.1109/CCAAW.2019.8904915

Downloaded from Cranfield Library Services E-Repository 